Introduction DNA repair enzymes could modulate the individual susceptibility to the genotoxic effect of exposure to ionising radiation (IR).

Methods The influence of polymorphisms of XRCC1, XRCC3 and XPD genes on the onset of chromosomal and DNA damage has been investigated in 43 workers exposed to low levels of IR, including 36 healthcare professionals and 7 industrial radiologists (exposed workers), and 43 subjects not occupationally exposed to IR (controls), matched for age. Chromosomal aberrations (CA) and micronuclei (MN) frequencies in peripheral blood lymphocytes were measured according to standard procedures and used as cytogenetic biomarkers, while Tail Intensity (TI) was the parameter of the Comet test used to evaluate oxidative DNA damage. Genotypic variants Arg194Trp, Arg280His and Arg399Gln for XRCC1, Thr241Met for XRCC3 and Lys751Gln for the XPD genes were analysed using the restriction fragment length polymorphism technique.

Results Both total CA and chromosome breaks frequencies were significantly higher in the exposed workers than controls $(\mathrm{p}<0.05$ and $\mathrm{p}<0.01$ respectively), while no significant differences between the two groups were observed in terms of chromatid breaks and MN frequencies as well as the TI. In the controls only, TI was significantly higher in females than males, whereas a smoking habit did not affect the biomarkers investigated. The genetic polymorphisms of XRCC1, XRCC3 and XPD, individually analysed, did not influence any of the genotoxicity and oxidative damage biomarkers studied, either in the exposed workers or the controls.

Discussion Chromosome breaks frequency resulted a valid cytogenetic biomarker for the monitoring of workers exposed to low doses of IR. The presence of single genetic variants reducing the activity of DNA repair enzymes does not seem to determine an increased risk of genotoxic effects of low doses of IR.

\section{EXPOSURE OF EMPLOYEES IN THE GERMAN TRANSPORT INDUSTRY TO SOLAR UV RADIATION}

Gabriele Meyer*, Christian Felten. German Social Accident Insurance Institution for Commercial Transport, Postal Logistics and Telecommunication (BG Verkehr), Hamburg, Germany

\subsection{6/oemed-2018-ICOHabstracts. 1226}

Introduction In Germany, the number of skin cancer diseases, which is caused by ultraviolet radiation (UV radiation) of sunlight, increases considerably. The exposure to solar UV radiation can be effected outdoors during a professional activity. But reliable data about the level of UV radiation exposure are missing.

In the research project 'skin cancer induced by UV radiation', initialized and realised by the German social accident insurance, the actual exposure should be identified under realistic working conditions. The objective is to create a taskrelated exposure matrix.

Methods From april till october 2015, UV radiation was determined in different groups of occupational activities. 18 people were involved, who were exclusively working at the airport ramp including handling agents (passenger handling), handling agents (cargo handling), turn round coordinators (TRC), turn round supervisors and gardeners. During their daily working hours they wore an electronic dosimeter, which is fixed at an arm mount and determines the erythemal exposure radiation. The data were recorded and anonymously transferred to a server.

Result During summer, the UV exposure of occupational groups is as different as their work. Compared to other occupational groups, apron workers are exposed to a medium level of solar UV radiation. Values between 129 and 322 SED were determined. Among the apron workers, the handling agents (passenger handling) get the highest erythemal exposure.

Discussion Sun protection has to be adapted to the expected UV exposure of the different occupational groups. The data can be used for the development of tailor-made prevention measures.

\section{SHORT-TERM RADON 222 MEASUREMENT MODELLING}

${ }^{1}$ Daniele Costantini, ${ }^{1,2}$ Enrico Marchetti. 'Sapienza University of Roma, Radiology Technician Degree School; ${ }^{2}$ talian National Institute for Insurance Against Accidents at Work, Department of Medicine, Epidemiology, Workplace and Environmental Hygiene, Monte Porzio Catone (RM), Italy

\subsection{6/oemed-2018-ICOHabstracts. 1227}

Introduction Radon concentration undergoes seasonal and daily changes. Those changes are kept into account by long-term measurement: in Italian law this period is one year. Following the excess of limits, it is mandatory to start mitigation activities. The assessment of the new concentration cannot exceed few weeks, otherwise it will infringe the want for workers' safety.

Methods The aim of present work is to assess feasibility of short-term measurement that can substitute longer ones. In order to do so we measured radon in the same room by two different methods one long-term integration (reliable) and another one that gives short-term time history. Radon concentration was assessed by half-yearly CR39 and active alfa spectrometer Tracerlab in the same room. The spectrometer daily curves were modelled by mathematical functions to fit changes while giving the CR39 integrated value.

Result After comparison of modelled values with integrated ones, it comes out that the very important step is to keep into account, in the modellization, the occupation and the use of rooms, along with their duration. If this step is accurate, the numbers are comparable with those of passive methods.

Discussion Essentially two occupational phases gives two functions to reproduce integrated values: a sinusoidal and an exponential function. The correct duration with the window opening/closing time profile, provide the estimation of values of parameters to be used with those functions.

This gives the coincidence of actual and foreseen data of daily radon changes, saving a whole lot of measuring time and, hence, money.

\section{RADIATION PROTECTION IN PUBLIC HOSPITALS IN ABIDJAN}

A Arnaud. Universite Felix Houphouet Boigny

10.1136/oemed-2018-ICOHabstracts. 1228

Introduction Advances in medical imaging have helped to better orient the therapeutic strategy and evaluate the 
effectiveness of treatments. The use of ionising radiation in radiology, however, is not without risks for health professionals directly involved in radiation work.

Methods The study was cross-sectional and prospective, including medical imaging technicians and engineers selected radiology departments, regularly hired and assigned to radiation work. Data were reported on a self-administered questionnaire.

Results Five hospitals were selected with 59 participants, including 6 (9\%) women, $54(92 \%)$ senior technicians and 5 $(8 \%)$ engineers. The cumulative age group of 30-50 years constituted $95 \%$ of the participants; 16 (27\%) participants had been exposed for 5-10 years, 2 (4\%) had been exposed for more than 20 years; The risk of cancer (96.6\%), radiodermatitis (31\%) and infertility (71.4\%) were recognised by the participants. The main PPEs identified by the participants as radio-protectors were the lead apron (96.6\%), the leaded glove $(68.6 \%)$, the shells $(31 \%)$, the anti-RX goggles $(57.8 \%)$; $33.2 \%$ of the participants wore them regularly, $60.6 \%$ were irregular, $6.2 \%$ did not wear them. PPE was available for $37.8 \%$ of the presentations. The interest of the dosimeter was known to $94.4 \%$ of the participants. Apparatus was revised in $19 \%$ of cases; $91 \%$ of participants received IR training and were qualified to work under radiation. Pictograms existed in $40 \%$ of hospitals, light signals in $80 \% ; 58.2 \%$ of the presentations knew their meaning.

Discussion The low availability and irregular wearing of PPE, and the ignorance of hazard indicators are more likely to expose them to IR.

Conclusion Strengthen protection measures through the availability of PPE and training

\section{OCCUPATIONAL SKIN CANCER IN OUTDOOR WORKERS IN ITALY: EXPECTED NUMBER VS CASES RECOGNISED BY THE ITALIAN NATIONAL COMPENSATION AUTHORITY (INAIL)}

${ }^{1}$ Alberto Modenese*, 'Leena Korpinen, ${ }^{1}$ Fabriziomaria Gobba. ${ }^{1}$ Department of Biomedical, Metabolic and Neural Sciences, University of Modena and Reggio Emilia, Modena, Italy; ${ }^{2}$ Clinical Physiology and Neurophysiology Unit, North Karelia Central Hospital and Honkalampi, Joensuu, Finland

\subsection{6/oemed-2018-ICOHabstracts.1229}

Introduction Solar Ultraviolet Radiation (UV) is one of the main risk factors for Non Melanoma Skin Cancer (NMSC) and Malignant Melanoma (MM). In Italy, only considering agriculture, fishery and construction sectors, the approximate number of workers exposed to solar UV (Outdoor workers OW-) is 2 million (1.6 million males, 400000 females).

Our aim is to compare the number of skin cancers (SCs) expected in OW to the number recognised by the Italian National Compensation Authority (INAIL).

Methods We collected data of Italian National Cancer Registres and the INAIL database of occupational diseases, including cancer, respectively available on the websites www. registri-tumori.it and www.bancadaticsa.inail.it

Results In Italy the yearly incidence of $\mathrm{MM}$ is 14,2 per 100000 in males and 13 per 100000 in females, that of NMSC 119.4 per 100000 in males and 90.7 per 100000 in females.
Applying these incidence rates to the Italian OW number, the expected SCs per year are approximately 2561 (279 MMs and 2282 NMSCs). INAIL recognised as occupational disease n. 246 cases (20\% MM vs $80 \%$ NMSCs) in the last 5 years, i.e. less than 50 cases per year.

Discussion and conclusions Our results show that, in Italy, the National Compensation Authority recognises less than the 2\% of the cases expected to occur in OW each year: 50 vs 2500. Main limitations of these data are that the incidence rates applied to OWs were not standardised, that the number of solar UV related SCs calculated is possibly under-estimated, considering that, e.g., not all OW groups were included, and that the data from cancer registries were quite outdated while the SCs incidence is increasing.

In conclusion, our data suggest a large under-estimation of occupational SCs in Italy, and that a better recognition of these diseases in OW is a relevant, and urgent, problem.

\section{GLIOMAS INCIDENCE IN ITALY}

${ }^{1}$ Alberto Modenese*, ${ }^{2}$ Leena Korpinen, ${ }^{1}$ Fabriziomaria Gobba. 'Department of Biomedical, Metabolic and Neural Sciences, University of Modena and Reggio Emilia, Modena, Italy; ${ }^{2}$ Clinical Physiology and Neurophysiology Unit, North Karelia Central Hospital and Honkalampi, Joensuu, Finland

\subsection{6/oemed-2018-ICOHabstracts.1230}

Introduction The International Agency for Research on Cancer has classified Radio Frequency in the Group 2B, 'possibly carcinogenic to humans', based on an association found between exposure related to mobile phones use and risk of gliomas and acoustic neuromas, even if this classification was discussed for some inconsistencies. Considering the large increase in mobile phone users from the ' $80 \mathrm{~s}$, an increase in incidence of these tumours should be expected. The aim of this work is to follow the incidence of gliomas in Italy.

Methods The national incidence of central nervous system (CNS) cancers and that of gliomas from 2006 was obtained from the Italian Cancer Registries

Result Considering CNS cancers, in the period 1996-2009 the yearly age-standardised incidence per 100000 has decreased from 10.8 to 9.8 in males, and from 8.0 to 7.0 in females. The incidence of gliomas, available for years 2006-2009 only, shows a slight increase from 5.7 to 6.3 in males, while is substantially stable (around 4) in females.

Discussion and conclusion Since the late '90 s, mobile phone use in Italy has largely increased: according to World Bank data, rose from 11.3 mobile phones per 100 Italians in 1996, to 107.7 in 2004 (exceeding the number of inhabitants), and 142.1 in 2015. If RF esposure related to mobile phones is associated with gliomas, considering a possible latency of 10 20 years an increase of the incidence of this tumours should be expected, at least from the second half of the 2000s

Considering all CNS tumours, during the period 19962009 data show a decreasing trend in Italy. For glioma, currently available standardised incidence rates, covering only the period 2006-2009, suggest a slight increase, but limited to males. Data available at this moment do not adequately support any firm conclusion on the trends of these tumours in Italy. 